\title{
A unique partnership between a health system's insurance plan and the Integrative Medicine Department benefits patients and helps control costs
}

\author{
Maureen M Anderson ${ }^{1}$, Karen Armstrong ${ }^{1}$, Katherine Nori Janosz ${ }^{2}$, Michael Tocco ${ }^{1}$, Nancy A DeVore ${ }^{3}$, Geoffrey \\ Stachura ${ }^{1}$, Linda Styczynski ${ }^{3}$, Karen Stachura ${ }^{3}$, Hallie Armstrong ${ }^{1}$, Gail Elliott Patricolo*1 \\ ${ }^{1}$ Integrative Medicine, Beaumont Health, United States \\ ${ }^{2}$ Division of Nutrition and Preventive Medicine, Beaumont Health, United States \\ ${ }^{3}$ Beaumont Employee Health Plan, Beaumont Health, United States
}

Received: January 29, 2016

Accepted: April 24, 2016

Online Published: May 6, 2016

DOI: $10.5430 /$ jha.v5n4p61

URL: http://dx.doi.org/10.5430/jha.v5n4p61

\begin{abstract}
Health care costs continue to increase, affecting patients and insurance providers. Complementary health approaches are increasingly used to augment traditional medicine, and integrative medicine (IM) incorporates these complementary approaches into traditional patient care. The IM Department was established in our institution in 2004 and now offers a wide range of services to patients. Our institution offers health care coverage to all benefit-eligible hospital personnel and their eligible dependents. The use of IM has had a surprising and beneficial effect on the health care costs of this small, self-insured health plan. We found that the coverage of certain IM modalities for specific conditions had positive clinical results and resulted in significant cost savings to the insurance plan. At the same time, this partnership supports patients by providing appropriate and effective care, and we have seen success in terms of patient recovery and patient satisfaction. Here, we present the history of the relationship between the insurance plan and the IM Department, how the coverage of IM modalities has expanded, and the current practice at our institution. We demonstrate that this innovative relationship has benefitted patients and resulted in cost-savings for the insurance provider. Therefore, this partnership will continue to expand, thus providing patients with a wide range of treatment options and effective care.
\end{abstract}

Key Words: Integrative medicine, Cost savings, Employee health insurance, Health care costs

\section{INTRODUCTION}

The increasing costs of health care affect both patients and insurance providers. ${ }^{[1,2]}$ As patients look to control their health care costs, complementary health approaches are increasingly used to augment traditional medicine. Integrative medicine (IM) incorporates these non-traditional techniques into traditional patient care. ${ }^{[3]}$ The use of these approaches has increased dramatically in recent years in the United States (US), with 33\% of Americans using at least one type of complementary health approach in $2012,{ }^{[4]}$ and worldwide. ${ }^{[5]}$ The IM Department was established at Beaumont Health in 2004 and now offers services such as clinical massage, acupuncture, yoga therapy, reflexology, cranial sacral therapy, integrative nutrition services, functional medicine, and

\footnotetext{
*Correspondence: Gail Elliott Patricolo; Email: gail.elliottpatricolo@ beaumont.org; Address: Integrative Medicine, Beaumont Health, 3577 W. Thirteen Mile Road Royal Oak, MI 48073, United States.
} 
naturopathic medicine. ${ }^{[6]}$ The success of the IM program is due not only to positive clinical results and patient demand but also to support from the hospital system, including administration, medical personnel, and the insurance provider. Beaumont Health offers health care coverage to all benefiteligible hospital personnel and their eligible dependents; this health care program is an employer self-insured health plan known as the Beaumont Employee Health Plan (BEHP). Although some countries mandate coverage of IM modalities, IM treatments are not typically covered by health insurance in the US. However, BEHP found that coverage of certain IM modalities for certain conditions would be cost-effective and benefit patients. Here, we discuss the unique relationship between BEHP and the IM Department. We will present the history of the relationship, how the coverage of IM modalities has expanded, and how this innovative partnership has benefitted patients and resulted in cost-savings for the insurance provider.

\section{IM AND THE EMPLOYEE HEALTH PLAN}

\subsection{Initiation and expansion}

The novel paradigm of including IM modalities as a covered benefit for patients was initiated in 2009 as a response to handle workers' compensation claims that involved musculoskeletal or neuromuscular pain. The medical director of Occupational Health Services (OHS), who is also the medical director for BEHP, met with the director of IM to discuss how IM modalities could help this patient population. The IM director recommended a treatment plan of one acupuncture session and one clinical massage session per week for eight weeks for patients with musculoskeletal or neuromuscular pain whose conditions were not resolved by traditional, allopathic care. Based on this recommendation, this treatment plan was authorized as a covered benefit under workers' compensation. This acupuncture and clinical massage regimen was immensely successful, in terms of patient recovery and allowing patients to return to work, and patients were pleased with having expanded treatment options.

Due to the success seen with the worker's compensation patients, the medical director initiated a trial program in which BEHP would provide for its members coverage of certain IM modalities for certain conditions. Therefore, starting in 2010 , the treatment plan that had shown results for worker's compensation claims was offered to all BEHP members $(35,000$ in 2010): acupuncture and clinical massage were offered as a covered benefit for musculoskeletal complaints (with a maximum of eight sessions each). In addition, oncology patients who were in active treatment and those who were up to two years post-treatment were offered acupuncture and clinical massage for any complaint. The IM Department charges \$60 for each massage session and \$112 for each acupuncture session. In conjunction with this new benefit, coverage for chiropractic services was reduced from 38 visits to 12 annually - a $32 \%$ reduction. This single change in coverage - covering IM services while reducing chiropractic services - resulted in a savings for BEHP of \$1 million (US dollars). This dramatic cost savings was accompanied by positive clinical results and increased patient satisfaction, based on unsolicited reports from patients regarding their improved health and quality of life (QoL). In addition, this change in coverage increased the number of domestic claims, while ensuring a high level of patient care.

Based on these results, coverage for other IM modalities began to be expanded. For example, in conjunction with the expanded coverage for musculoskeletal conditions, later in 2010, naturopathic medicine consultations were also covered as an additional benefit to oncology patients at a cost of $\$ 180$ per appointment. In addition, all patients with non-structural headaches and migraines were eligible to receive up to three naturopathic medicine consults. These changes were patientand results-driven, as members of the IM Department and BEHP met regularly to evaluate patient conditions and needs and to determine how IM modalities could benefit patients with certain conditions.

Initially, the coverage for the IM services was considered a trial program and was not widely advertised but instead relied on word of mouth and physician referral. In 2012, based on the success of the collaboration to date, the program expanded. At this time, the list of covered IM services was included in the BEHP plan benefit summary, thus greatly expanding the number of patients utilizing these services. In addition, the number of diagnoses approved for IM services continued to expand. For example, in 2012, naturopathic medicine consults (three consults annually) and acupuncture (eight sessions) were included as an additional covered benefit for all BEHP members who were diagnosed with migraines, allergies, insomnia, infertility, and gastrointestinal issues. With such positive results for members utilizing the services of the IM Department, in 2013, BEHP member benefits also expanded to the Division of Nutrition and Preventive Medicine. This division provides personalized and unique treatment plans directed at optimizing patient health by offering individualized and group exercise sessions, nutrition appointments and classes, and group and individual psychology sessions.

\subsection{Current practice}

Figure 1 provides the timeline of how IM modalities were incorporated into the benefit package for BEHP members. Currently, clinical massage, acupuncture and naturopathic 
medicine consultations are covered services for a number of medical diagnoses. Table 1 indicates the IM options available for a number of different conditions and indicates which treatments are currently covered benefits for BEHP members. BEHP members are required to pay a co-pay of $\$ 20$ per visit for IM services. Musculoskeletal conditions, such as joint or muscle pain in the body including but not limited to the neck, low back, shoulder, hip or knee, arthritis and fibromyalgia are covered for clinical massage and acupuncture. Cancerrelated side effects and chronic headaches can be covered for clinical massage, acupuncture and naturopathy. Gastrointestinal issues, such as gastroesophageal reflux disease, irritable bowel syndrome, indigestion, constipation or diarrhea, res- piratory issues, such as asthma, sinusitis and allergies, male and female infertility support, insomnia and sleep disturbances, among others can be treated by acupuncture and naturopathic medicine. In addition, consultations with integrative/functional medicine physicians (board-certified in IM or Holistic Medicine) are covered. These integrative physicians spend a greater amount of time with their patients than allopathic physicians can, thus allowing them to more fully understand the patient's social and mental history and physical lifestyle and allowing the patient to be treated in a holistic manner - as a whole person instead of a set of symptoms.

$\begin{array}{llll}\begin{array}{c}2009- \\ \text { Workers' } \\ \text { Compensation }\end{array} & \begin{array}{c}\text { Early 2010- } \\ \text { Pilot for } \\ \text { BEHP }\end{array}\end{array}$

Figure 1. The evolution of the partnership between the IM Department and BEHP

Table 1. A partial list of conditions seen in the IM Department and the IM modalities that can be used for treatment

\begin{tabular}{lccc}
\hline & Clinical Massage & Acupuncture & Naturopathic Medicine \\
\hline ADHD & & $\mathrm{C}$ & $\mathrm{C}$ \\
Allergies/asthma & $\mathrm{C}$ & $\mathrm{C}$ & $\mathrm{C}$ \\
Arthritis pain & $\mathrm{C}$ & $\mathrm{C}$ & $\mathrm{X}$ \\
Fibromyalgia & $\mathrm{C}$ & $\mathrm{C}$ & $\mathrm{C}$ \\
Headaches & & $\mathrm{C}$ & $\mathrm{C}$ \\
IBS/Gastrointestinal issues & & $\mathrm{C}$ & $\mathrm{C}$ \\
Infertility & & $\mathrm{C}$ & $\mathrm{C}$ \\
Insomnia & $\mathrm{C}$ & $\mathrm{C}$ & $\mathrm{C}$ \\
Musculoskeletal pain & $\mathrm{C}$ & $\mathrm{C}$ & $\mathrm{X}$ \\
Oncology-related conditions & $\mathrm{C}$ & $\mathrm{C}$ & $\mathrm{C}$ \\
Pelvic pain & $\mathrm{X}$ & $\mathrm{X}$ & $\mathrm{C}$ \\
Post-operative scar therapy & & & \\
Tinnitus & & & \\
\hline
\end{tabular}

Note. "X" indicates that the listed IM modalities can be used to treat that condition, but are not currently covered by BEHP; "C" indicates the listed IM modalities that are also covered benefits for that diagnosis; ADHD: attention-deficit/hyperactivity disorder; BEHP: Beaumont Employee Health Plan; IBS: irritable bowel syndrome; IM: integrative medicine

Preauthorization by BEHP for treatment in the IM Department is required for coverage. Prescriptions for an IM service are not accepted by the IM practitioners or BEHP. Documentation of the condition by a medical practitioner, such as a chart note stating the diagnosis or condition (e.g., "migraines" or "back pain"), is required. This requirement ensures that the patient has been examined and properly diagnosed by a medical physician. Preauthorization for treatment is given for a specific regimen for a specific period of time. For example, currently, the number of covered acupuncture and 
clinical massage sessions has increased to 12 sessions each for one authorization, which must be obtained yearly. For patients that require additional treatment sessions, a plan of care is required. This plan of care is a treatment plan that is generated by the IM practitioners for each member requiring extended treatments. This plan details the condition and patient progress to date and outlines future treatment plans for the patient. After approval by the IM medical director, the plan is shared with BEHP, who evaluates the treatment plan and determines approval for the recommended sessions.

This authorization and treatment process allows for treatment flexibility, as services can be individualized for each patient and their condition. As mentioned, IM practitioners treat the patient holistically; thus, each patient may require a different treatment regimen, even for the same diagnosis. In addition, the chart notes provide detailed information regarding the patient's medical history. The IM providers can use this information to determine the treatment plan that is optimal for each individual patient, which may require additional IM services not initially recommended. In some cases, patients may be referred to other departments, such as workability, the weight control center, physical therapy, etc. This plan of care process allows for this personalization, while still providing oversight and accountability.

\subsection{Challenges}

Initiating this partnership between BEHP and the IM Department encountered certain challenges related to the fact that this service is unique, as IM modalities are not typically covered by insurance in the US, although coverage varies by state. Thus, program initiation and expansion occurred slowly to allow integration into the medical system. In addition, communicating to medical professionals the coverage available to their patients and the benefits to their patients of using IM services was necessary to ensure full participation.

Another challenge is that plan members are often unaware of the coverage for IM services, even though the services are listed in the plan benefit summary. To counter this situation, a brochure was developed outlining the IM services available and, specifically, which medical diagnoses are covered for each IM modality. Furthermore, in general, patients are ill-equipped with regard to navigating their ever-changing benefit coverage. Patients may comply with the treatment plan outlined by their primary physician, but they may not take the extra time to identify which other services are open to them. To counter this situation, hospital physicians must be educated regarding the IM services available and the coverage options for BEHP members. In addition, care must be coordinated between allopathic and IM practitioners, which requires open and thorough communication.
Finally, an additional challenge has been, and continues to be, staffing and space for the IM Department. The number of appointments in the IM Department for BEHP members has increased yearly from 2010-2013, with a plateau noted from 2013-2015, reflecting the capacity limits of the department (see Figure 2; the numbers do not include physician appointments). In addition, the number of covered diagnoses for IM services continues to expand, thus increasing the number of eligible patients and the potential demand for IM services. In 2015, the number of covered lives for the BEHP program was approximately 35,000; however, this number is expected to increase to $>55,000$ in 2017 , due to recent affiliations. The hospital system and the IM Department must match the anticipated increase in demand by increasing staffing and space to provide services, while also caring for patients in the community who are not BEHP members.

\subsection{Advantages}

The advantages of this partnership have been immense to both patients and the health care insurance provider. Patients show positive clinical results and receive the support and care that they need with treatment plans individualized for their unique situations. In addition, patients love this partnership - the use of complementary health services is increasing in general, and patients are thrilled that they can utilize these services and feel empowered with regard to their health, while keeping their out-of-pocket costs reasonable. IM services are considered financially conservative, safe, non-invasive treatments. Patients are typically eager to attempt to manage their health care needs through the use of IM services before turning to invasive surgery or high-cost pharmaceuticals that may have substantial side effects. Due to HIPAA regulations, it is prohibited by law for an insurance provider to track the number of days off for any individual. However, anecdotally, patients have self-reported a reduced number of days off of work due to chronic problems, such as migraines. In addition, we have documented that a substantial number of patients are able to reduce or eliminate their opioid intake, including sleep aid medication, with the use of IM services.

The financial benefits to BEHP have been surprisingly strong. The third-party administrator for the health plan reported that, in 2014, the costs related to musculoskeletal conditions decreased by $19.7 \%$ compared with prior years. This result is in addition to the $\$ 1 \mathrm{M}$ savings noted earlier that was realized after adjusting chiropractic coverage in 2010. In addition, while the prevalence of patients with lower back pain showed a slight decrease of $3.8 \%$, the costs related to the treatment of lower back pain decreased by $19.5 \%$ from 2013 to 2014. These savings can be attributed to the use of IM services, as this coverage was the only change to the plan 
during these time frames. Traditional therapies are still being used to treat musculoskeletal conditions and lower back pain; however, as the use of IM modalities has increased, the cost to the plan has decreased. This finding is supported by other studies that have reported improved results and cost savings with the use of IM. ${ }^{[7-11]}$ Lind et al. reported in 2010 that, in Washington State, which does mandate coverage of IM modalities by health care providers, patients who used complementary health approaches to deal with back pain, fibromyalgia, or menopause had significantly lower health care expenditures than non-users. ${ }^{[8]}$ In a study across the US, Martin et al. also found that users of complementary health approaches had lower costs associated with back and neck pain. ${ }^{[9]}$ These reports support our findings that covering IM modalities for musculoskeletal conditions and lower back pain reduced health expenditures for these conditions.

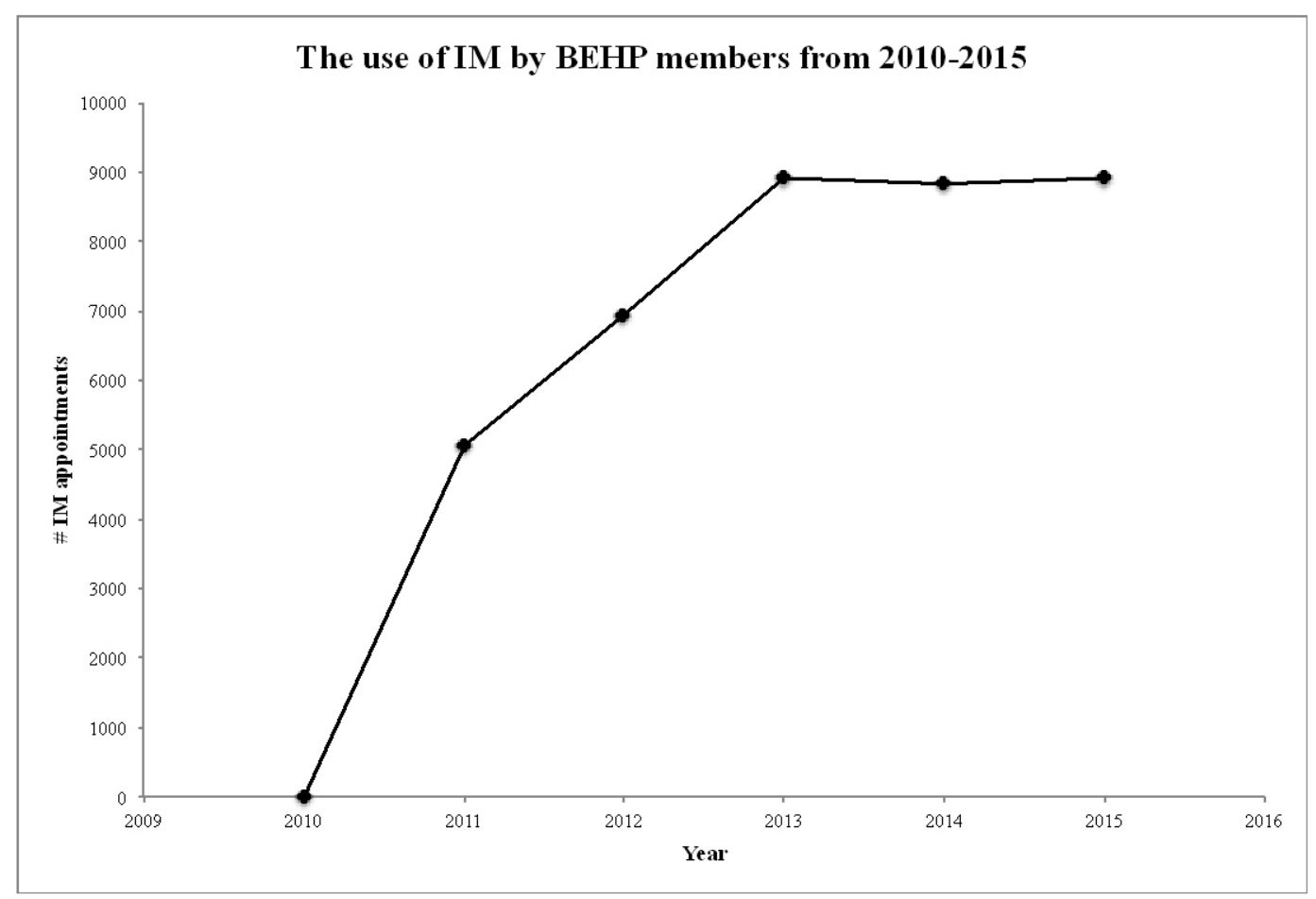

Figure 2. The number of appointments for BEHP members in the IM Department from 2010-2015 (does not include physician appointments)

Another benefit to including IM services in the health care plan has been to increase the number of domestic claims, while reducing the number of outside claims. In addition to the financial benefits of keeping patients within the system, there are benefits to the patients as well. Keeping patients within the hospital system ensures that patients are receiving care that is appropriate for their medical situation from providers who are highly trained and qualified. In addition, keeping patients within the system allows for the results to be monitored through the use of electronic medical records and for the treatment regimen to be altered if the patient is not progressing. Finally, the patients report positive clinical results and improved QoL to the IM practitioners and to the nursing staff at BEHP. The patients overall feel better supported, as there is improved communication between medical specialties and departments, which is very difficult when the patient receives outside care.

Published by Sciedu Press

\subsection{Future plans}

Our future plans are to continue to provide comprehensive support to patients, while expanding the collaboration between BEHP and the IM Department to increase research on IM modalities. Several pilot research studies are either being planned or are in progress. One such study involves investigating the effects of acupuncture and clinical massage on chronic headaches and migraines. Anecdotal evidence indicates that a treatment plan of several acupuncture and clinical massage sessions can have significant positive effects on chronic headaches and migraines, which can severely impact patient QoL, work performance, and absenteeism. Another topic of interest involves the use of acupuncture, naturopathic medicine, and functional medicine to address chronic inflammatory conditions. These conditions not only negatively affect patient QoL but also incur high costs to BEHP related to treatment. Again, anecdotal evidence in- 
dicates that these IM modalities can dramatically improve these conditions, and formal research studies are needed to quantify and verify these effects.

Supporting patients also involves providing coordinated care across medical specialties and providers within the system. In addition, we wish to help patients learn to manage and control their own health. Health coaches and health psychologists can guide individuals and/or groups through education sessions on proper diet, exercise, relaxation, etc. Group support has been shown to be a useful method to help patients make lifestyle changes and achieve their health goals. ${ }^{[12]}$ Similarly, shared medical appointments may be implemented in the future. In these appointments, a group of patients with similar needs attend the same appointment. While specific medical conditions are discussed privately, the patients can hear presentations as a group from different specialists (e.g., integrative nutrition experts, acupuncturists, yoga therapy, mindfulness techniques, naturopathic medicine doctors, etc.). These types of appointments benefit both the medical system, which has reduced costs under this paradigm, and patients, who benefit from the group environment, as they are able to speak with many different specialists and learn to manage their health in a holistic manner.

IM services are expected to continue to expand. As stated, recent affiliations are expected to increase the number of covered lives to $>55,000$ in 2017. Therefore, the IM Department must also expand, and there are plans to increase the number of IM physicians and other practitioners on staff to accommodate the increased demand. In addition, coverage for IM modalities is also expected to expand. There are certain advanced laboratory testing techniques, such as advanced micronutrient testing, that are not currently considered standard but which can provide useful information to medical care practitioners. These advanced tests may be covered in the future. Likewise, the IM Department has its own pharmacy that stocks certain pharmaceutical-grade herbs and supplements that are not currently covered by BEHP, but

\section{REFERENCES}

[1] Patton M. U.S. Health Care Costs Rise Faster Than Inflation. 2015 [updated Jun 29, 2015; cited 2015 Oct 23, 2015]. Available from: http://www.forbes.com/sites/mikepatton/2015/06/29 /u-s-health-care-costs-rise-faster-than-inflation/

[2] Pear R. Health Insurance Companies Seek Big Rate Increases for 2016. New York Times. 2015 [updated July 3, 2015; cited 2015 Oct 23, 2015]. Available from: http://www.nytimes.com/2015/07/ 04/us/health-insurance-companies-seek-big-rate-inc reases-for-2016.html?_r=0 which may be a covered benefit in the future.

\section{Conclusions}

The use of complementary health approaches continues to increase in the US and worldwide. ${ }^{[5]}$ While some countries provide health care coverage of IM modalities, most states in the US do not. However, our experiences found that coverage of IM modalities for certain conditions benefits patients and the health care insurer. We have documented improvements in patient conditions, and patients report improved QoL with the use of IM services. In addition, patients are pleased with the option of using IM modalities to address their health needs before resorting to invasive procedures or pharmaceutics that may have adverse side effects. Authorizing patients to use IM services has resulted in dramatic cost savings to the health care insurance plan by increasing domestic claims and through reducing patient medication use. Increasing domestic claims also improves patient care by allowing for continuity of care across departments within the system and by ensuring that patients receive high quality care. In addition, this integration of care across departments allows patients to be treated in a holistic manner, improving patient care and satisfaction. Based on the success of this innovative partnership to date, this relationship will continue and be strengthened. Research studies involving IM modalities and specific health conditions are in progress, and additional IM services may be covered in the future, based on treatment effectiveness and patient demand. Finally, our experience indicates that the thoughtful coverage of certain IM modalities for specific conditions is beneficial to both the patient and to the health care insurer, and this paradigm represents a novel, strategic approach to health care.

\section{ACKNOWLEDGEMENTS}

We would like to thank Christine Billecke, Ph.D. for her assistance in the preparation of this manuscript.

\section{CONFlicts OF INTEREST Disclosure}

The authors declare they have no conflict of interest.
[3] Kiefer D, Pitluk J, Klunk K. An overview of CAM: components and clinical uses. Nutrition in clinical practice: official publication of the American Society for Parenteral and Enteral Nutrition. 2009; 24(5): 549-59. http://dx.doi.org/10.1177/0884533609342437

[4] Clarke TC, Black LI, Stussman BJ, et al. Trends in the use of complementary health approaches among adults: United States, 2002-2012. National health statistics reports. 2015; 79: 1-16. PMid: 25671660.

[5] Frass M, Strassl RP, Friehs H, et al. Use and acceptance of complementary and alternative medicine among the general population and medical personnel: a systematic review. Ochsner J. 2012; 12(1): 
45-56. PMid: 22438782.

[6] Lanni T, Patricolo GE. The business of integrative medicine in a large hospital system. Journal of Hospital Administration. 2014; 3(3): 52-60. http://dx.doi.org/10.5430/jha.v3n3p52

[7] Lafferty WE, Tyree PT, Bellas AS, et al. Insurance coverage and subsequent utilization of complementary and alternative medicine providers. Am J Manag Care. 2006; 12(7): 397-404. PMid: 16834526.

[8] Lind BK, Lafferty WE, Tyree PT, et al. Comparison of health care expenditures among insured users and nonusers of complementary and alternative medicine in Washington State: a cost minimization analysis. J Altern Complement Med. 2010; 16(4): 411-7. http://dx.doi.org/10.1089/acm.2009.0261
[9] Martin BI, Gerkovich MM, Deyo RA, et al. The association of complementary and alternative medicine use and health care expenditures for back and neck problems. Med Care. 2012; 50(12): 1029-36. http://dx.doi.org/10.1097/MLR.0b013e318269e0b2

[10] Herman PM, Poindexter BL, Witt CM, et al. Are complementary therapies and integrative care cost-effective? A systematic review of economic evaluations. BMJ open. 2012; 2(5). http://dx. doi . org /10.1136/bmjopen-2012-001046

[11] Milano C. Less pain, lower costs: can integrative medicine do both? Manag Care. 2012; 21(3): 38-40. PMid: 22471164.

[12] Paturel A. Power in numbers: Research is pinpointing the factors that make group therapy successful. Monitor on Pyschology. 2012: 48. http://dx.doi.org/10.1037/e652122012-023 